\title{
Fragility of the spin-glass-like collective state to a magnetic field in an interacting Fe-
} C nanoparticle system

\author{
Jönsson, P. E.; Felton, S.; Svedlindh, P.; Nordblad, P.; Hansen, Mikkel Fougt
}

Published in:

Physical Review B

Link to article, DOI:

10.1103/PhysRevB.64.212402

Publication date:

2001

Document Version

Publisher's PDF, also known as Version of record

Link back to DTU Orbit

Citation (APA):

Jönsson, P. E., Felton, S., Svedlindh, P., Nordblad, P., \& Hansen, M. F. (2001). Fragility of the spin-glass-like collective state to a magnetic field in an interacting Fe-C nanoparticle system. Physical Review B, 64(21), 212402. https://doi.org/10.1103/PhysRevB.64.212402

\section{General rights}

Copyright and moral rights for the publications made accessible in the public portal are retained by the authors and/or other copyright owners and it is a condition of accessing publications that users recognise and abide by the legal requirements associated with these rights.

- Users may download and print one copy of any publication from the public portal for the purpose of private study or research.

- You may not further distribute the material or use it for any profit-making activity or commercial gain

- You may freely distribute the URL identifying the publication in the public portal 


\title{
Fragility of the spin-glass-like collective state to a magnetic field in an interacting $\mathrm{Fe}-\mathrm{C}$ nanoparticle system
}

\author{
P. E. Jönsson, S. Felton, P. Svedlindh, and P. Nordblad \\ Department of Materials Science, Uppsala University, Box 534, SE-751 21 Uppsala, Sweden \\ M. F. Hansen \\ Department of Physics, Building 307, Technical University of Denmark, DK-2800 Lyngby, Denmark
}

(Received 26 June 2001; published 2 November 2001)

\begin{abstract}
The effect of applied magnetic fields on the collective nonequilibrium dynamics of a strongly interacting $\mathrm{Fe}-\mathrm{C}$ nanoparticle system has been investigated. It is experimentally shown that the magnetic aging diminishes to finally disappear for fields of moderate strength. The field needed to remove the observable aging behavior increases with decreasing temperature. The same qualitative behavior is observed in an amorphous metallic spin glass $\left(\mathrm{Fe}_{0.15} \mathrm{Ni}_{0.85}\right)_{75} \mathrm{P}_{16} \mathrm{~B}_{6} \mathrm{Al}_{3}$.

DOI: 10.1103/PhysRevB.64.212402

PACS number(s): 75.50.Tt, 75.50.Lk, 75.10.Nr, 75.50.Mm
\end{abstract}

Frozen ferrofluids offer systems where the long-range dipolar interaction between the single-domain nanoparticles can be continuously varied by changing the particle concentration. In a dilute ferrofluid, where the dipolar interaction energy is negligible compared to the anisotropy energy, the magnetic properties of the system are given by averaging over the individual particle contributions. The dynamical properties of isolated particles are determined by the thermally activated relaxation between the potential wells of the anisotropy energy, as was originally proposed by Néel. ${ }^{1}$ The magnetic response of a single-domain particle is strongly affected by an external bias field. The relaxation time depends both on the magnitude and the direction of the applied magnetic field with respect to the anisotropy axis, in combination with the damping of the gyration around the easy axis after a spin flip (see, e.g., Refs. 2-5). For interacting particle systems, the dipolar field created by surrounding particles will also affect the relaxation. ${ }^{6,7}$

Highly concentrated ferrofluids, in which the dipolar interaction energy dominates over the anisotropy energy, contain all ingredients needed to create collective glassy dynamics; a complex interaction mechanism-the dipolar interaction-and frustration provided by the randomness of the particle positions and directions of the anisotropy axes. Indeed, experiments have shown that such particle systems exhibit nonequilibrium dynamics with striking similarities to the nonequilibrium dynamics of spin glasses. The magnetic relaxation of the low field dc magnetization shows an aging behavior $^{8}$ and there is a downward relaxation of the lowfrequency ac susceptibility when the sample is kept at constant temperature combined with a rejuvenation and memory behavior when the temperature of the sample is further decreased and subsequently reheated. ${ }^{9,10}$ Also, certain effects of applied magnetic fields on the nonequilibrium behavior of particle systems have been reported. In a recent work it was shown that the relaxation of the thermoremanent magnetization exhibits magnetic aging if the field applied during cooling is low, but not if a relatively high field is used. ${ }^{11}$ This result is similar to the behavior of spin glasses where largeenough fields impose an equilibrium state on experimental time scales and a relaxation of the thermoremanent magne- tization that is independent of wait time ${ }^{12}$ and it is thus also consistent with the assumption of collective spin-glass-like dynamics in strongly interacting nanoparticle systems. ${ }^{13}$ In the spin-glass case, it has also been shown that field changes can induce rejuvenation of the spin-glass state, ${ }^{14}$ and that the nonequilibrium relaxation is affected by large-enough fields. $^{15,16}$

In this paper, we study the effect of bias-magnetic fields on the nonequilibrium dynamics of a strongly interacting nanoparticle sample. We have chosen to measure the relaxation of the low-frequency ac susceptibility, since such relaxation is indicative of magnetic aging and, therefore, does not exist in weakly interacting nanoparticle systems. It is observed that the collective nonequilibrium dynamics disappears at moderate fields and that the strength of the field needed to remove the collective glassy dynamics increases with decreasing temperature. The same qualitative behavior is observed for a spin-glass sample. We interpret the results within the droplet model, ${ }^{17}$ which is a real-space model that has successfully been used to describe nonequilibrium effects in spin glasses.

The ferrofluid consisted of ferromagnetic nanoparticles of the amorphous alloy $\mathrm{Fe}_{1-x} \mathrm{C}_{x}(x \approx 0.2-0.3)$. A TEM study revealed a nearly spherical particle shape and a particle size of $d=5.3 \pm 0.3 \mathrm{~nm}$. The saturation magnetization is estimated to $M_{s}=1 \times 10^{3} \mathrm{G}$ and the anisotropy constant to $K$ $=9 \times 10^{5} \mathrm{erg} \mathrm{cm}^{-3}$. Details about the sample preparation and characterization are given elsewhere. ${ }^{18}$ Two samples were studied, one with a concentration of $5 \mathrm{vol} \%$ that has earlier been shown to exhibit spin-glass-like nonequilibrium dynamics, ${ }^{10}$ while the second sample was diluted to $1 \mathrm{vol} \%$ and no magnetic aging was observed in this sample.

For comparison, experiments were also performed on an amorphous metallic spin glass $\left(\mathrm{Fe}_{0.15} \mathrm{Ni}_{0.85}\right){ }_{75} \mathrm{P}_{16} \mathrm{~B}_{6} \mathrm{Al}_{3}$. The interaction in this sample is of Ruderman-Kittel-KasuyaYosida type and it behaves as a dilute metallic spin-glass alloy with a transition temperature of $T_{g}=22.5 \mathrm{~K}$. Various magnetic properties of the system have been investigated in a number of earlier reports (see, e.g., Refs. 19, 20, 14, and 15). We chose this compound since it has a high susceptibility, and comparably low applied magnetic fields are needed to 
affect the nonequilibrium dynamics.

The temperature dependence of the ac susceptibility of the two nanoparticle samples with a superimposed dc field was measured in a LakeShore 7225 ac susceptometer using an ac probing field of frequency $125 \mathrm{~Hz}$ and amplitude 2 Oe and dc fields in the range $0-250$ Oe. These fields are low enough not to destroy the two-well structure of the single-particle potential. $^{21}$ A noncommercial low-field superconducting quantum interference device magnetometer ${ }^{22}$ was used to measure the ac susceptibility with superimposed dc fields as a function of time on both the 5 vol\% nanoparticle sample and the spin glass. The samples were cooled in the dc field from a temperature in the paramagnetic region to the measuring temperature, and the data collection was initiated after waiting a short time $t_{0}$ allowing the system to stabilize. For the nanoparticle sample $t_{0}=120 \mathrm{~s}$ and for the spin glass $t_{0}$ $=60 \mathrm{~s}$. The frequency of the ac field was $510 \mathrm{mHz}$, the field amplitude $10 \mathrm{mOe}$ and the range of dc fields 0-240 Oe.

The total energy of a nanoparticle system, probed by an ac field of amplitude $h_{0}$ and with an applied dc field $H$ in the same direction as the ac field, is given by

$$
E=E_{\mathrm{a}}-\sum_{i} \vec{m}_{i} \cdot\left(h_{0} \hat{z} \sin \omega t+H \hat{z}+\vec{H}_{i}^{\mathrm{dip}}\right),
$$

where $E_{a}$ is the anisotropy energy and $\vec{m}_{i}$ is the magnetic moment of particle $i$. The dipolar field at the position of the $i$ th particle is given by

$$
\vec{H}_{i}^{\mathrm{dip}}=\sum_{j}\left[\frac{3\left(\vec{m}_{j} \cdot \vec{r}_{i j}\right) \vec{r}_{i j}}{r_{i j}^{5}}-\frac{\vec{m}_{j}}{r_{i j}^{3}}\right],
$$

where $j \neq i$ and $\vec{r}_{i j}=\vec{r}_{i}-\vec{r}_{j}$ is the vector connecting particle $i$ with particle $j$.

The results from measurements of the ac susceptibility with superimposed dc fields for the two nanoparticle samples are shown in Fig. 1. For $H=0$ the peak of the ac susceptibility appears at higher temperature for the $5 \mathrm{vol} \%$ sample than for the $1 \mathrm{vol} \%$ sample, due to the stronger dipolar interactions in the more concentrated sample. However, the difference between the susceptibility of the two samples decreases with increasing dc field, and for the highest fields the susceptibility curves are almost identical indicating that the dipolar field created by the surrounding particles is negligible compared to the applied field. Corresponding acsusceptibility results with bias fields for the spin-glass sample are shown in Fig. 1 of Ref. 20.

We have chosen to study how the collective behavior is affected by a magnetic field by measuring the relaxation of the low-frequency ac susceptibility in superimposed dc fields. Aging effects are seen in $\chi^{\prime \prime}(\omega)$ as a relaxation towards equilibrium with time spent at constant temperature, $t$ $(\gg 1 / \omega) .{ }^{23}$ The corresponding relaxation is seen in $\chi^{\prime}(\omega)$, and since the relaxation is larger in $\chi^{\prime}$ than in $\chi^{\prime \prime}$ it can be more convenient to study $\chi^{\prime}$ if the relaxation is small. ${ }^{10} \mathrm{Fig}$ ure 2 shows $\chi(H, t)-\chi\left(H, t=t_{0}\right)$ for different dc fields at $T=25 \mathrm{~K}$ for the $5 \mathrm{vol} \%$ sample. It is seen that the field reduces the relaxation and at fields higher than 200 Oe there is almost no relaxation left. A reduction of the relaxation in
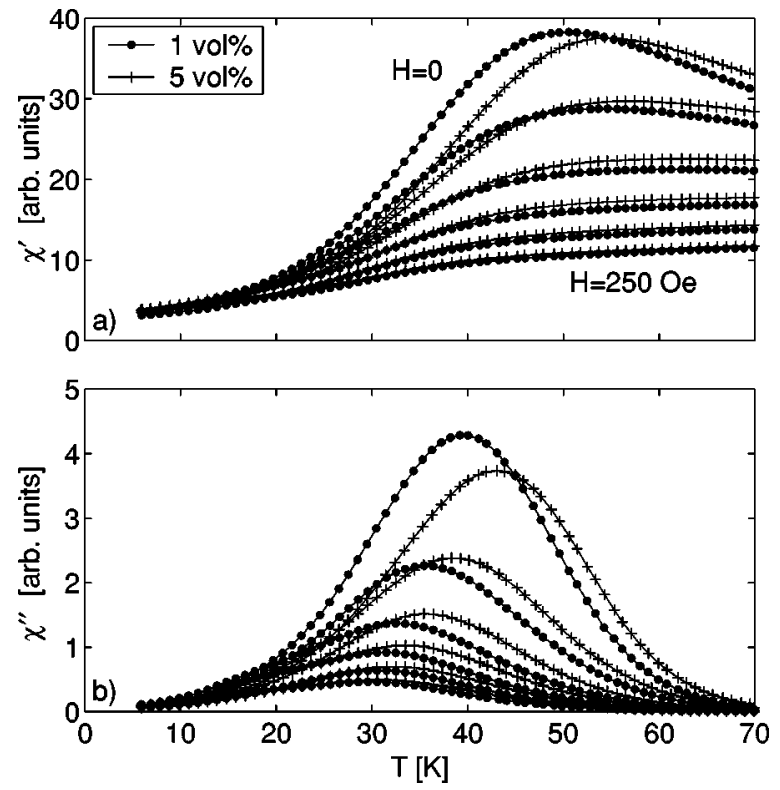

FIG. 1. Ac susceptibility vs temperature for different superimposed dc fields; $H=0,50,100,150,200,250$ Oe. $f=125 \mathrm{~Hz}$.

the ac susceptibility with applied magnetic fields is also observed for the spin-glass sample.

We now define a quantity $k$ as

$$
k(H)=\frac{\chi\left(H, t=t_{0}\right)-\chi\left(H, t=t_{\max }\right)}{\chi\left(H=0, t=t_{0}\right)-\chi\left(H=0, t=t_{\max }\right)},
$$

which gives a relative measure of the relaxation in the presence of a dc field. We have repeated the measurements in Fig. 2 at different temperatures for both the $5 \mathrm{vol} \%$ sample

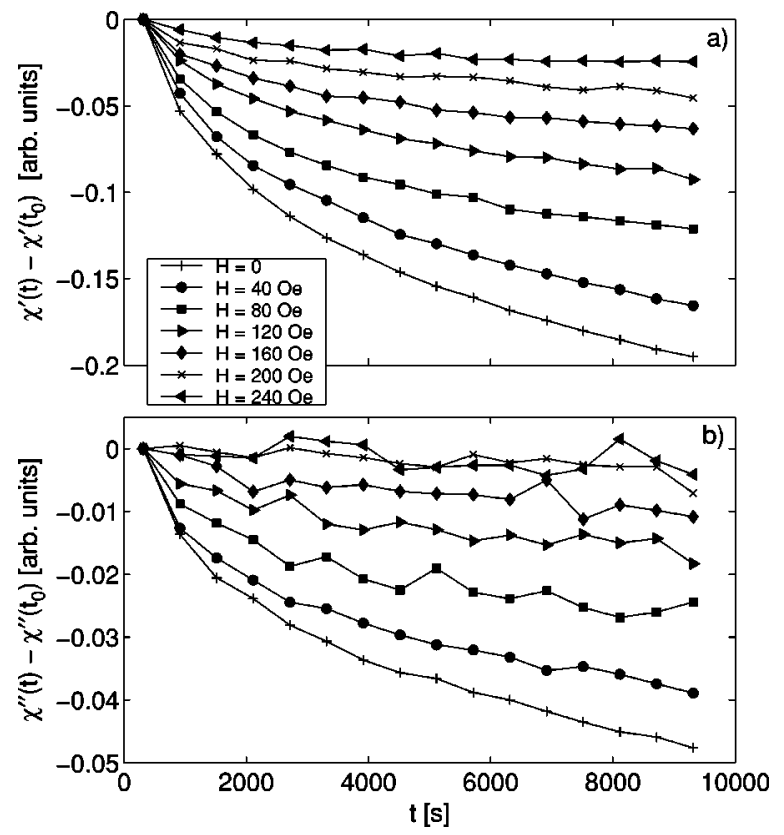

FIG. 2. Relaxation of the ac susceptibility for different superimposed dc fields $H$, measured on the 5 -vol\% nanoparticle sample. The frequency $\omega / 2 \pi=510 \mathrm{mHz}$ and the temperature $T=25 \mathrm{~K}$. Same units as in Fig. 1. 


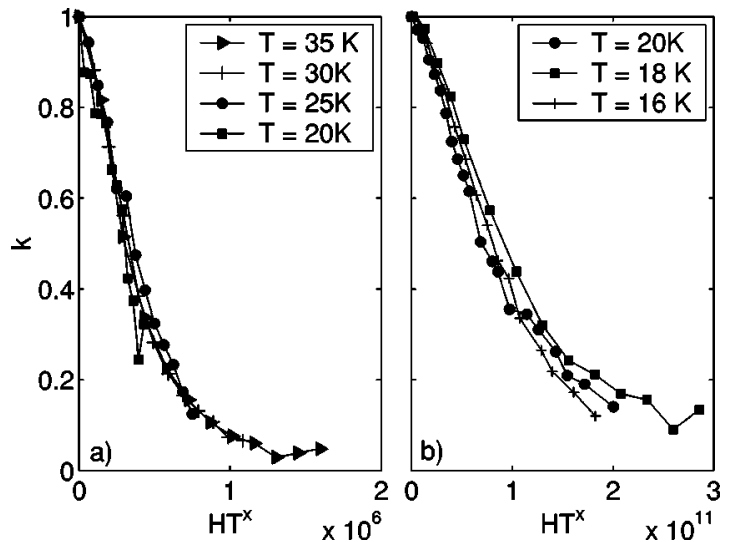

FIG. 3. Quantity $k\left(H T^{x}\right)$ at different temperatures for (a) the 5 -vol\% nanoparticle sample using $x=2.5$ and (b) the spin glass using $x=7.5$.

and the spin-glass sample. For both samples the relaxation persists to higher fields at lower temperatures. Postulating an $H T^{x}$ dependence of $k$ and using $x$ as a fitting parameter, we obtain reasonable scaling behavior for the two samples. In Fig. 3(a) $k\left(H T^{2.5}\right)$ is shown for temperatures in the interval 20-35 K, for the 5-vol\% nanoparticle sample. The curves measured at different temperatures give a satisfactory data collapse. For the spin-glass sample, on the other hand, data collapse is obtained for curves measured at different temperatures between 16 and $20 \mathrm{~K}$ using $k\left(H T^{7.5}\right)$, as can be seen in Fig. 3(b). The choice of using $\chi^{\prime}$ or $\chi^{\prime \prime}$ in the analysis did not affect $k$.

We will interpret our results within the droplet model $^{17}$ that was derived for short-range Ising spin glasses. Important concepts of the model should, however, also be applicable to particle systems exhibiting strong dipole-dipole interaction and random orientation of the anisotropy axes. ${ }^{10}$ In this model, it is assumed that, at each temperature below the spin-glass transition temperature $T_{g}$, the spin-glass equilibrium state is unique but twofold degenerate by its global spin-reversal state. In equilibrium, the most important contribution to physical observables, such as the magnetic susceptibility, comes from low-lying excitations of compact clusters of spins, called droplets. The droplet excitations of size $L$ have a broad distribution of free energies, with a typical value of

$$
F_{L}^{\mathrm{typ}} \sim \mathrm{Y}\left(L / L_{0}\right)^{\theta},
$$

where $Y(T)$ is the stiffness modulus, $\theta$ is the stiffness exponent, and $L_{0}$ is a characteristic length scale. The stiffness exponent satisfies $\theta \leqslant(d-1) / 2$, where $d$ is the dimension of the system, and $\theta \approx 0.2$ for $d=3 .^{24,25}$ The dynamics of droplets is a thermally activated process. The typical energy barrier scales as

$$
B_{L}^{t y p} \sim \Delta(T)\left[L / L_{0}\right]^{\psi},
$$

where $\Delta(T)$ sets the free-energy scale of the barriers and the barrier exponent $\psi$ satisfies $\theta \leqslant \psi \leqslant d-1$. The value of the exponent $\psi$ has been estimated to be $0.8 .^{26}$
Let us now consider the isothermal aging process that we have observed experimentally as a relaxation of the lowfrequency ac susceptibility. Within the droplet model, the development towards equilibrium from the out-ofequilibrium state, which was created when quenching the system, is governed by the growth of domains belonging to either of the two degenerate equilibrium states. This growth is driven by successive nucleation and annihilation of droplets. The growth law proposed by Fisher and Huse ${ }^{17}$ is

$$
L_{T}(t) \sim L_{0}\left[\frac{T \ln \left(t / \tau_{0}\right)}{\Delta(T)}\right]^{1 / \psi},
$$

where $\tau_{0}$ is the relaxation time of a spin (or magnetic moment). The weak ac field of frequency $\omega / 2 \pi$ probes the system by polarizing droplets of size $L_{T}(1 / \omega)$. Since $t \gg 1 / \omega$, $L_{T}(1 / \omega)<L_{T}(t)$ and hence the domain walls of size $L_{T}(t)$ appear effectively frozen on the probing length scale. The small-scale droplets [of size $L_{T}(1 / \omega)$ ] in touch with a frozen-in domain wall will have a lower free energy than they would have had if the frozen-in wall was not present. This can be described by a reduction of the effective stiffness of the system. Fisher and Huse used scaling arguments to obtain

$$
\frac{\Delta \Upsilon}{\Upsilon} \sim\left[\frac{L_{T}(1 / \omega)}{L_{T}(t)}\right]^{d-\theta}
$$

and deduced that

$$
\chi^{\prime \prime}(\omega)=\chi_{e q}^{\prime \prime}(\omega)\left\{1-c\left[\frac{L_{T}(1 / \omega)}{L_{T}(t)}\right]^{d-\theta}\right\},
$$

where $c$ is a constant. It has been shown experimentally, for a two-dimensional Ising spin glass, that both $\chi^{\prime \prime}$ and $\chi^{\prime}$ relax according to this expression. ${ }^{27}$

In a magnetic field $H$, the system is disordered by the field on length scales larger than the correlation length

$$
\xi_{H} \sim\left[\frac{\Upsilon}{H \sqrt{q_{m}}}\right]^{2 /(d-2 \theta)},
$$

while it still exhibits spin-glass order on shorter length scales. Here, $q_{m}(T)$ is an order parameter defined in Ref. 17. The typical time needed for the system to equilibrate is given by $t_{e q} \sim \tau_{H}$, where $\ln \left(\tau_{H} / \tau_{0}\right) \sim(\Delta / T) \xi_{H}^{\psi}$. The relaxation of $\chi$ at a certain temperature will then be governed by the relation between the domain size $L_{T}(t)$ reached within the experimental time window and the length scale $\xi_{H}$ set by the magnetic field. In a strong applied field, Eq. (5) can be modified to include the correlation length as

$$
\chi^{\prime \prime}(\omega, H)=\chi_{e q}^{\prime \prime}(\omega, H)\left\{1-c\left[\frac{L_{T}(1 / \omega)}{\min \left(L_{T}(t), \xi_{H}\right)}\right]^{d-\theta}\right\} .
$$

Here, it should be noted that neither $L_{T}(t)$ nor $\xi_{H}$ are well defined length scales, so the relaxation will not end abruptly, but will gradually be suppressed over a wide time window. Three different field regimes can be distinguished: (i) $L_{T}(t)$ $\ll \xi_{H}$, the collective nonequilibrium dynamics is virtually unaffected by the field; (ii) $L_{T}(t) \lesssim \xi_{H}$, the system is partly 
at equilibrium, and hence the ac relaxation is reduced; and (iii) $L_{T}(t)>\xi_{H}$, the system is in equilibrium, no collective dynamics exists.

At sufficiently low temperatures $\left(T / T_{g} \lesssim 0.5\right)$ the influence of critical fluctuations is small and hence the temperature-dependent coefficients $Y, \Delta$, and $q_{m}$ are approximately constant, while they vanish at $T_{g} \cdot{ }^{17}$ Since $k(H)$ scales with $L_{T}(t) / \xi_{H}$ according to Eq. (6), we then expect to obtain data collapse, at low temperatures, plotting $k$ vs $H T^{\tilde{x}}$, with $\tilde{x}=(d-2 \theta) / 2 \psi$. The reported values of $\theta$ and $\psi$ yield $\tilde{x} \approx 1.6$ and hence the condition $L_{T}(t) / \xi_{H} \approx 1$ is fulfilled for lower fields at higher temperatures in accordance with results shown in Fig. 3.

For the spin-glass sample $x=7.5$ was obtained [see Fig. 3(b)]. Due to experimental limitations, these measurements correspond to $T / T_{g} \sim 0.7-0.9$ and it is expected that $x \neq \tilde{x}$ since close to the transition critical fluctuations will modify the temperature dependence. The larger value of $x$ than of $\tilde{x}$ indicates that the temperature dependence of the field needed to affect the collective dynamics is stronger close to $T_{g}$ than at lower temperatures. This is consistent with how a bias field affects the ac susceptibility vs temperature curve (see Fig. 1, Ref. 20) - the ac susceptibility is the most affected by bias fields close to $T_{g}$.
For the nanoparticle sample, $x=2.5$ was obtained [see Fig. 3(a)]. This apparent better agreement with the estimated value of $\tilde{x}$ does not mean that the particle system is better described by the droplet model than the studied isotropic spin glass. For the nanoparticle sample one may, e.g., expect some further discrepancy due to the relaxation time of an individual magnetic moment. Unlike the spin-flip time of an atomic moment in a spin glass that is constant, the relaxation time of a magnetic moment depends both on the temperature and the field in a nontrivial way, and is also affected by the dipolar interactions.

We have shown, by measuring the isothermal relaxation of the ac susceptibility with superimposed dc fields, that the collective glassy dynamics of a strongly interacting nanoparticle system can be destroyed by the application of moderate fields. The field strength needed to destroy the collective dynamics increases with decreasing temperature. This behavior is consistent with corresponding observations on a spinglass sample. The results for both samples show qualitative agreement with predictions within the droplet model.

We thank J. L. García-Palacios, H. Yoshino, and H. Takayama for valuable discussions. This work was financially supported by the Swedish Natural Science Research Council (NFR), now assimilated in the Swedish Research Council (VR).
${ }^{1}$ L. Néel, Ann. Geophys. (C.N.R.S.) 5, 99 (1949).

${ }^{2}$ W. F. Brown, Jr., Phys. Rev. 130, 1677 (1963).

${ }^{3}$ A. Aharoni, Phys. Rev. 177, 793 (1969).

${ }^{4}$ D. A. Garanin, E. C. Kennedy, D. S. F. Crothers, and W. T. Coffey, Phys. Rev. E 60, 6499 (1999).

${ }^{5}$ W. T. Coffey, D. A. Garanin, H. Kachkachi, and D. J. McCarthy, J. Magn. Magn. Mater. 221, 110 (2000).

${ }^{6}$ D. V. Berkov and N. L. Gorn, J. Phys.: Condens. Matter 13, 9369 (2001).

${ }^{7}$ P. E. Jönsson and J. L. García-Palacios, Europhys. Lett. 55, 418 (2001).

${ }^{8}$ T. Jonsson, J. Mattsson, C. Djurberg, F. A. Khan, P. Nordblad, and P. Svedlindh, Phys. Rev. Lett. 75, 4138 (1995).

${ }^{9}$ H. Mamiya, I. Nakatani, and T. Furubayashi, Phys. Rev. Lett. 82, 4332 (1999).

${ }^{10}$ P. Jönsson, M. F. Hansen, and P. Nordblad, Phys. Rev. B 61, 1261 (2000).

${ }^{11}$ M. García del Muro, X. Batlle, and A. Labarta, Phys. Rev. B 59, 13584 (1999).

${ }^{12}$ P. Granberg, P. Svedlindh, P. Nordblad, L. Lundgren, and H. S. Chen, Phys. Rev. B 35, 2075 (1987).

${ }^{13}$ P. Jönsson and P. Nordblad, Phys. Rev. B 62, 1466 (2000).

${ }^{14}$ C. Djurberg, P. Granberg, J. Mattsson, and P. Nordblad, Phys. Rev. B 50, 15775 (1994).
${ }^{15}$ C. Djurberg, J. Mattsson, and P. Nordblad, Europhys. Lett. 29, 163 (1995).

${ }^{16}$ Y. G. Joh, R. Orbach, and J. Hammann, Phys. Rev. Lett. 77, 4648 (1996).

${ }^{17}$ D. Fisher and D. A. Huse, Phys. Rev. B 38, 373 (1988); 38, 386 (1988).

${ }^{18}$ M. F. Hansen, P. Jönsson, P. Nordblad, and P. Svedlindh, cond-mat/0010090 (unpublished).

${ }^{19}$ P. Svedlindh, L. Lundgren, P. Nordblad, and H. S. Chen, Europhys. Lett. 2, 805 (1986).

${ }^{20}$ P. Svedlindh, L. Lundgren, P. Nordblad, and H. S. Chen, Europhys. Lett. 3, 243 (1987).

${ }^{21}$ The critical field (in the longitudinal case) is given by $H_{c}$ $=2 K / M_{S} \sim 1800$ Oe.

22 J. Magnusson, C. Djurberg, P. Granberg, and P. Nordblad, Rev. Sci. Instrum. 68, 3761 (1997).

${ }^{23}$ L. Lundgren, P. Svedlindh, and O. Beckman, J. Magn. Magn. Mater. 25, 33 (1981).

${ }^{24}$ A. J. Bray and M. A. Moore, J. Phys. C 17, L463 (1984).

${ }^{25}$ T. Komori, H. Yoshino, and H. Takayama, J. Phys. Soc. Jpn. 68, 3387 (1999).

${ }^{26}$ J. Mattsson, T. Jonsson, P. Nordblad, H. Aruga Katori, and A. Ito, Phys. Rev. Lett. 74, 4305 (1995).

${ }^{27}$ A. G. Schins, A. F. M. Arts, and H. W. de Wijn, Phys. Rev. Lett. 70, 2340 (1993). 